ISSN: 1980-055X

Recebido em: 13/04/2010

Aceito para publicação em: 25/07/2010

\title{
ESTIMATIVA DA TEMPERATURA DE SUPERFÍCIE EM ÁREAS URBANAS EM PROCESSO DE CONSOLIDAÇÃO: REFLEXÕES E EXPERIMENTO EM PLANALTINA-DF
}

\author{
Valdir Adilson Steinke ${ }^{8}$ \\ Ercília Torres Steinke ${ }^{9}$ \\ Carlos Hiroo Saito ${ }^{10}$
}

\begin{abstract}
RESUMO
Este trabalho teve como objetivo analisar um dos procedimentos de extração das informações de temperatura de superfície com base em imagens de satélite da série Landsat. Para tanto foi selecionada a bacia hidrográfica do Rio Monteiro, na qual está situada a cidade de Planaltina, no Distrito Federal. Todos os procedimentos metodológicos foram realizados em software de processamento de imagem digital. Os resultados indicaram a necessidade de avançar em pesquisas nesta temática devido à dinâmica dos procedimentos e em função da complexidade dos elementos envolvidos.
\end{abstract}

Palavras-Chave: Sensoriamento Remoto, Urbanização, Climatologia.

\begin{abstract}
This study aimed to analyze one of the procedures for extraction of information from surface temperature, based on satellite imagery of the Landsat series. Was chosen for this, the Rio Monteiro basin, where the city of Planaltina is located, in Distrito Federal. All the methodological procedures were performed with digital image processing software. The results indicated the need for progress in research on this topic due to the dynamic of the procedures and the complexity of the involved elements.
\end{abstract}

Key-words: Remote Sensing, Urbanization, Climatology.

\footnotetext{
${ }^{8}$ Geógrafo, Dr. em Ecologia, Professor Adjunto no Depto. de Geografia e Pesquisador Associado no Laboratório de Climatologia Geográfica-LCGea da Universidade de Brasília-UnB.

9 Geógrafa, Dra. em Ecologia, Professora Adjunta no Depto. de Geografia e Coordenadora do Laboratório de Climatologia Geográfica-LCGea da Universidade de Brasília-UnB.

${ }^{10}$ Biólogo, Dr. em Geografia, Professor Associado no Depto. de Ecologia da Universidade de BrasíliaUnB.
} 


\section{INTRODUÇÃO}

A transformação dos espaços pela consolidação e transformação das cidades, com a substituição de áreas de vegetação natural e espaços rurais em áreas impermeabilizadas, originaram um fenômeno climático em que a temperatura média costuma ser mais elevada do que nas regiões rurais próximas. (ARNFIELD, 2003; OKE, 1982, 1997; VOOGT, 2002).

Nas cidades nas quais os índices de densidade populacional tendem a elevar-se em função de seu crescimento populacional desproporcional ao crescimento de área, a inserção de atividades antrópicas de alto impacto e a elevada concentração urbana contribuem significativamente com a intensificação desse fenômeno pela construção de pavimentos de concreto e asfalto e edificações de toda ordem, convertendo a superfície em um "ladrilho" impermeabilizante do solo. Bonan (2002) chama a atenção para o calor excessivo produzido nas cidades e emitido para a atmosfera de modo contínuo, transformando as áreas urbanas em verdadeiros condutores locais para oscilações significativas na variabilidade climática, com conseqüências negativas para as sociedades que ocupam estes espaços.

A compreensão e quantificação dos fatos geradores desse fenômeno, mais conhecido como ilha de calor, e como estes aspectos podem ser analisados tornam-se fundamentais na busca de melhorias da qualidade de vida de quem habita os ambientes urbanos (Grimm et al. 2008: Wu 2008a).

Quando se trata de bacias hidrográficas urbanas, a alteração da temperatura de superfície, e as conseqüências que essa alteração pode causar na temperatura do ar influenciam diretamente os processos envolvidos no sistema hidrológico de uma região. Entre esses processos podem ser citados, o aumento do volume de água drenado em períodos chuvosos, o que provoca enchentes, erosão das margens, assoreamento e escorregamento. Além disso, devido à impermeabilidade causada pela intervenção antrópica, o escoamento superficial adquire maior velocidade alterando a dinâmica de recarga dos aqüíferos.

Com o surgimento das geotecnologias e, mais especificamente do sensoriamento remoto, que ganha força no início da década de 1970 com o lançamento do primeiro satélite da série Landsat MSS, surgem novas possibilidades de apoio a análise climática, principalmente com o despontar de técnicas promissoras desenvolvidas no final dos anos da década de 1980 . Em se tratando da climatologia brasileira, um trabalho pioneiro e de grande importância foi o de Lombardo (1985), que identificou as ilhas de calor na cidade de São Paulo.

A partir do estudo de Lombardo (1985), que pode ser visto como o impulsionador desta linha de análise, outros estudos foram desenvolvidos. Porém, considerando-se que, nos dias de hoje, as imagens de satélite estão muito mais acessíveis acredita-se que sua aplicação na climatologia urbana ainda seja limitada.

O desenvolvimento tecnológico na área de geotecnologias ocorreu de forma rápida levando-se em conta que nas últimas quatro décadas a quantidade limitada de instrumentos orbitais saltou para um universo de dezenas de satélites e centenas de imagens que cobrem diferentes faixas do espectro eletromagnético e diferentes dimensões e escalas territoriais. 
No que diz respeito à utilização de imagens de satélite para os estudos de temperatura de superfície, a faixa do espectro maís utilizada é a do termal, o qual capta a radiação emitida pelas superfícies. No entanto, estes estudos ainda possuem uma difusão limitada na climatologia em função das limitações decorrentes da presença da atmosfera entre o alvo (superfície) e o sensor. (MENDONÇA, 2003; COLLISCHONN, 1998).

Dados provenientes de diversos sensores, resoluções mais altas e novas técnicas de processamento têm, recentemente, aperfeiçoado a precisão do sensoriamento remoto permitindo novas aplicações. A utilização de dados de satélites para estimar propriedades físicas da superfície e suas variações foi investigada, por exemplo, por CARNAHAN e LARSON (1990), que utilizaram dados do satélite LANDSAT TM 5 (canal termal), em meso escala, para analisar as diferenças de temperatura entre as áreas urbanas e rurais em Indianápolis; KIM (1992) estudou as ilhas de calor em Washington/DC apontando o significado do albedo dos solos e da disponibilidade de vapor d'água para o balanço energético da superfície; NICHOL (1994) elaborou uma metodologia para estudo do clima urbano em Singapura e defendeu a aplicabilidade dos dados termais do satélite LANDSAT TM 5, desde que se utilize correções relativas à emissividade, para avaliação das temperaturas de superfície; PETKOV et al. (1996) realizaram uma comparação entre as temperaturas do ar coletadas em estações meteorológicas e as temperaturas estimadas através do sensor AVHRR a bordo do satélite NOAA 11 para a região da Toscana, indicando que os dados NOAA-AVHRR podem fornecer informações sobre a variabilidade espacial da temperatura do ar. Outros exemplos podem ser encontrados em THOMAS (2002), e WENG (2004).

Vários autores, entre eles, KERR et al. (1992) e KERR et al. (2004) acreditam que o sensoriamento remoto tem possibilidade de contribuir com os estudos relacionados ao campo térmico das cidades (temperatura de superfície), sendo necessário que o sensor escolhido conte com uma resolução espectral que o habilite à captação da radiação emitida pelos corpos terrestres. Essa emissão ocorre na forma de ondas longas.

A radiação de ondas longas está situada segundo MENESES (2001), FERREIRA (2002) e FLORENZANO (2002) dentro do intervalo espectral que vai de 5,0 a $1000 \mu \mathrm{m}$, definindo essa extensão de comprimento de ondas como região do infravermelho termal do espectro eletromagnético (tabela 1). Essa região é conhecida como termal porque, segundo MENEZES (2001), o que se detecta no sensor é a radiação emitida pelos objetos, cuja intensidade é função da sua temperatura de superfície. A melhor faixa que permite uma maior transmissão da energia emitida pela Terra que alcança o sensor, nesta região espectral, é o intervalo de 8,0 a $14,0 \mu \mathrm{m}$ para imageamento orbital.

Vale lembrar que o fluxo energético emitido pela superfície e captado pelo sensor tem suas características modificadas por vários fatores tais como, perturbações promovidas pelo próprio sensor, incluindo a resolução radiométrica e dinâmica dos dados; pelos efeitos devidos à presença da atmosfera e, também, pelo perfil do relevo terrestre. Segundo ZULLO JUNIOR (1994) e MACHADO et al. (2003), o conhecimento destes fatores é de grande importância para que seja possível distinguir as variações relacionadas com a captura dos dados pelos satélites e das variações referentes às próprias grandezas medidas. 
Tabela 1: espectro de ondas eletromagnéticas em ordem crescente de comprimento de onda.

\begin{tabular}{|c|c|}
\hline Tipo de radiação & Comprimento de onda \\
\hline Raios Gama & $0.0001 \mathrm{~nm}-0.1 \mathrm{~nm}$ \\
\hline Raios X & $0.01 \mathrm{~nm}-100 \mathrm{~nm}$ \\
\hline Ultravioleta & $6 \mathrm{~nm} \mathrm{e}-380 \mathrm{~nm}$ \\
\hline Luz visível - Violeta & $380 \mathrm{~nm}-430 \mathrm{~nm}$ \\
\hline Luz visível - Azul & $430 \mathrm{~nm}-470 \mathrm{~nm}$ \\
\hline Luz visível - Azul esverdeado & $470 \mathrm{~nm}-500 \mathrm{~nm}$ \\
\hline Luz visível - Verde & $500 \mathrm{~nm}-560 \mathrm{~nm}$ \\
\hline Luz visível - Amarelo & $560 \mathrm{~nm}-600 \mathrm{~nm}$ \\
\hline Luz visível - Laranja & $600 \mathrm{~nm}-640 \mathrm{~nm}$ \\
\hline Luz visível - Vermelho Claro & $640 \mathrm{~nm}-710 \mathrm{~nm}$ \\
\hline Luz visível - Vermelho escuro & $710 \mathrm{~nm}-780 \mathrm{~nm}$ \\
\hline Infravermelho & 780 nm - 1 mm ( milímetro) \\
\hline Microondas & $1 \mathrm{~mm}-30 \mathrm{~cm}$ \\
\hline Ondas de rádio, $T V$, radar, etc.. & $1 \mathrm{~mm}-60 \mathrm{Km}$ \\
\hline
\end{tabular}

Fonte: NOVO (1998)

Entre esses efeitos, os de caráter atmosférico são os mais comumente corrigidos quando se trata de extração das temperaturas de superfícies por meio de satélites. Os principais efeitos observados nas imagens, devido à presença real da atmosfera entre o satélite e a superfície terrestre são, de acordo com ZULLO JUNIOR (1994), a diminuição da faixa de valores digitais possíveis registrados pelo sensor, diminuição do contraste entre superfícies adjacentes e alteração do brilho de cada ponto da imagem. Os gases (principalmente vapor d'água, oxigênio, ozônio e dióxido de carbono) e os aerossóis absorvem e espalham a radiação solar desde quando ela atinge a atmosfera até quando a deixa, depois de refletida pelo solo.

A presença da atmosfera perturba a transmissão da radiação solar em seu meio, modificando as intensidades de energia que chegam diretamente à superfície terrestre e ao satélite. Esta atenuação na iluminação da superfície terrestre e no sinal registrado pelo satélite é contrabalançada pela contribuição de outros tipos de radiação incidente e refletida que surgem com a presença da atmosfera. As perturbações causadas pela atmosfera devem-se, principalmente, à atuação dos processos de absorção e espalhamento na trajetória da radiação solar em direção à superfície da Terra e no caminho da radiação refletida para o satélite. (MIRALLES, 1991 e ZULLO JUNIOR, 1994).

De acordo com KERR et al. (2004), devido à essas limitações, a utilização de estimativas confiáveis constitui-se em um ponto crucial para qualquer estudo que envolva temperatura de superfície. O autor destaca, ainda, que além dos efeitos atmosféricos é preciso considerar os efeitos relativos à emissividade ${ }^{11}$ da superfície.

PRICE (1983) ressalta que esses efeitos parecem ser particularmente pronunciados em áreas urbanas localizadas em regiões de clima tropical onde a absorção e a emitância da radiação infravermelha pelo vapor d'água atmosférico

\footnotetext{
${ }^{11}$ A emissividade, definida como razão entre a excitância (densidade do fluxo radiante em uma superfície) de um material e a excitância de um corpo negro à mesma temperatura é de extrema importância quando se trabalha com dados de sensoriamento remoto na região do infravermelho termal. (NOVO, 1998)
} 
podem atribuir uma diferença de até $10^{\circ} \mathrm{C}$ entre a temperatura real da superfície e a estimada pelo satélite. Dessa forma, embora os valores de radiância da imagem possam ser convertidos em temperatura do corpo negro, usando a Lei de Planck, esta técnica subestima a temperatura de superfície, caso não sejam realizadas correções atmosféricas e de acordo com a emissividade de cada cobertura.

Estudos relacionados com a extração da temperatura de superfície por meio de satélite foram iniciados com a utilização de dados provenientes dos sensores AVHRR (Advanvced Very-High Resolution Radiometer) a bordo dos satélites NOAA (National Oceanic and Atmospheric Admnistration) administrados pelo National Environmental Satellite and Information Service (NESDIS) ${ }^{12}$. Os sensores contam com dois canais de detecção de energia eletromagnética emitida na faixa do infravermelho termal. Esses canais compreendem as regiões que vão de 10,3 a $11,3 \mu \mathrm{m}$ (canal 4) e 11,5 a 12,5 $\mu \mathrm{m}$ (canal 5). Os dados obtidos desses canais revelam especificamente as características termais da superfície da Terra, dos oceanos e dos topos das nuvens.

Os satélites da série NOAA foram projetados para operarem em uma órbita polar e heliossíncrona, ou seja, cada satélite passa nas regiões com o sol em uma posição aproximada a da passagem anterior, para qual o eixo maior possui $7.231,9 \mathrm{Km}$, formando um campo de observação de pontos do globo compreendidos entre $78^{\circ}$ de Latitude Norte e $78^{\circ}$ de Latitude Sul. Cada órbita possui um período de aproximadamente 102 minutos. Portanto, em um dia, 0 satélite faz em torno de 14 órbitas ao redor do planeta (1440 minutos divididos por 102 minutos por órbita). Atualmente, os satélites NOAA 17 e 18, desta série, fazem a cobertura do globo terrestre em uma área imageada de $2.400 \mathrm{~km}$, com resolução espacial de $1,1 \mathrm{~km}$ captando informações sobre uma mesma área seis vezes por dia (três diurnas e três noturnas).

Cada um dos atuais satélites da série NOAA possui um número de diferentes sensores com propósitos específicos, contudo, o único usado para fins meteorológicos é o AVHRR com resolução espacial de $1,1 \mathrm{~km}$ (nadir). Além dos dois canais já comentados anteriormente, este sensor possui mais três descritos na tabela 2.

O fato de o sistema NOAA apresentar uma boa resolução temporal aliada à baixa resolução espacial de seus produtos torna as imagens oferecidas pelo sistema aptas à estimativa da temperatura de superfície dentro de curtos intervalos de tempo, porém, EHRLICH et al. (1994) observaram que essas são características apreciáveis no desenvolvimento de estudos somente em escalas regionais.

Características do campo térmico de cidades foram inicialmente examinadas com a utilização de sensores de baixa resolução espacial tais como o AVHRR do satélite NOAA. Este foi utilizado por BALLING e BRAZEL (1988) e ROTH et al. (1989) para a identificação de ilhas de calor. LOMBARDO (1985) encontrou diferenças de nove graus de temperatura entre o centro da cidade de São Paulo e a periferia da mancha urbanizada em um trabalho pioneiro no Brasil utilizando imagens do satélite NOAA para o tratamento das condições térmicas e imagens do satélite LANDSAT para a identificação do uso da terra. A análise da

12 A família de satélites NOAA, administrada pelo NESDIS, foi iniciada em 1960 com os satélites da série TIROS e realizou o lançamento de mais de uma dezena de satélites e diversos instrumentos operacionais (sondas e imageadores). Esta série gera diariamente observações globais de padrões meteorológicos e condições ambientais na forma de dados quantitativos. 
termografia da superfície urbana permaneceu bastante genérica naquele estudo, uma vez que a resolução espacial das imagens NOAA não permitiu o detalhamento necessário que a análise climática local demanda.

Tabela 2: Canais do sensor AVHRR

\section{Canais do sensor AVHRR}

\begin{tabular}{|c|c|c|}
\hline Canal & $\begin{array}{l}\text { Resolução Espectral } \\
(\mu \mathrm{m})\end{array}$ & Tipos de dados obtidos \\
\hline 1 (visível) & $0,58-0,68$ & $\begin{array}{l}\text { Cobertura de nuvens, neve, gelo, } \\
\text { mapeamento, poluição. }\end{array}$ \\
\hline 2(IR próximo) & $0,725-1.10$ & $\begin{array}{l}\text { Cobertura de nuvens, superfícies } \\
\text { de água, vegetação. }\end{array}$ \\
\hline 3 (IR médio) & $3,55-3,93$ & $\begin{array}{l}\text { Cobertura de nuvens à noite, } \\
\text { temperatura do mar, incêndio e } \\
\text { vulcões. }\end{array}$ \\
\hline 4 (IR termal) & $10,3-11,3$ & $\begin{array}{l}\text { Cobertura de nuvens (dia e noite) } \\
\text { e padrões de temperatura da } \\
\text { Terra e mar. }\end{array}$ \\
\hline 5 (IR termal) & $11,5-12,5$ & $\begin{array}{l}\text { Vapor d'água, cobertura de } \\
\text { nuvens e temperaturas. }\end{array}$ \\
\hline
\end{tabular}

Fontes: adaptado de FERREIRA (2000) e ALMEIDA (1995)

DOUSSET e GOURMELON (2003) propuseram uma metodologia para analisar a variação espacial e temporal das temperaturas de superfície e sua relação com a cobertura da terra, em Los Angeles, por meio da combinação de dados oriundos do sensor AVHRR do satélite NOAA e do sensor High Resolution Visible - HRV do satélite Spot. A pesquisa demonstrou a aplicabilidade da combinação de dados termais (resolução de $1 \mathrm{~km}$ ) e dados do visível e do infravermelho próximo (resolução de $20 \mathrm{~m}$ ) para estudos de climatologia urbana.

Vários autores, entre eles, NICHOL (1994); WENG et al. (2004); MENDONÇA e DUBREUIL (2005); acreditam que as imagens provenientes do satélite LANDSAT, embora este seja um satélite de recursos naturais e não meteorológico como o NOAA, encontram uma aplicação no domínio da climatologia local por meio da utilização do canal infravermelho termal (banda 6) do sensor TM (Thematic Mapper), que apresenta uma resolução espacial de $120 \mathrm{~m}$. Segundo estes autores, estas imagens possibilitam a observação da variação térmica de superfície intra-urbana, tanto de grandes cidades, como as de menor porte, bem como da variação térmica na área rural.

Os satélites LANDSAT foram desenvolvidos pela National Aeronautics and Space Administration - NASA para a observação dos recursos terrestres tendo sido lançado em julho de 1972 o LANDSAT 1 (ERST - 1). Nos três primeiros satélites da série, o principal sistema sensor era o Multiespectral Scanner System (MSS), que operava em quatro canais (dois no visível e dois no infravermelho próximo), com uma resolução espacial de 80 metros. Os LANDSAT 1, 2 e 3 passavam sob a mesma área da superfície terrestre a cada 18 dias. (NOVO, 1998).

A partir do LANDSAT 4, lançado em 1982, além do MSS, foi colocado em operação um novo sistema sensor com tecnologia mais avançada, o Thematic 
Mapper (TM). Este sensor registra dados em 7 canais (três no visível, um no infravermelho próximo, dois no infravermelho médio e um no infravermelho termal) com uma resolução espacial de 30 metros (exceto para o termal - 120 metros). O LANDSAT 5, com as mesmas características do seu antecessor, foi lançado em 1984 e operou até recentemente. (FLORENZANO, 2002).

O Landsat 6 foi lançado em outubro de 1993, mas não conseguiu atingir sua órbita e foi declarado perdido. O LANDSAT 7 foi lançado em abril de 1999, no qual o sensor TM foi substituído pelo $\mathrm{ETM}^{+}$(Enhanced thematic mapper, plus). A principal diferença foi a Adição de uma banda pancromática com resolução espacial de $15 \mathrm{~m}$. As bandas do infravermelho visível mantiveram a resolução espacial de $30 \mathrm{~m}$ (bandas $1,2,3,4,5,7$ ) e as bandas do infra vermelho termal (bandas $6 \mathrm{~L}$ e $6 \mathrm{H}$ ) passam a ser adquiridas com resolução de 60 metros (tabela 3). Atualmente está em funcionamento o LANDSAT 7 que completa 0 ciclo de imageamento da Terra a cada 16 dias. Cada imagem obtida por este satélite cobre uma área de 185 por $185 \mathrm{Km}$. A tabela 3 mostra os sensores e suas respectivas faixas espectrais do satélite LANDSAT.

Tabela 3. Canais dos sensores do satélite LANDSAT

\begin{tabular}{|c|c|c|}
\hline Canais & $\begin{array}{l}\text { Resolução espectral } \\
(\mu \mathrm{m})\end{array}$ & $\begin{array}{c}\text { Resolução } \\
\text { espacial }\end{array}$ \\
\hline $\begin{array}{l}1(\mathrm{TM} \mathrm{e} \\
\left.\mathrm{ETM}^{+}\right)\end{array}$ & $0,4-0,5$ & $30 \mathrm{~m}$ \\
\hline $\begin{array}{l}2\left(\mathrm{TM}^{\mathrm{e}}\right. \\
\left.\mathrm{ETM}^{+}\right)\end{array}$ & $0,5-0,6$ & $30 \mathrm{~m}$ \\
\hline $\begin{array}{l}3\left(\mathrm{TM} \mathrm{e}^{\mathrm{e}}\right. \\
\left.\mathrm{ETM}^{+}\right)\end{array}$ & $0,63-$ & $30 \mathrm{~m}$ \\
\hline $\begin{array}{l}4\left(\mathrm{TM}^{\mathrm{e}}\right. \\
\left.\mathrm{ETM}^{+}\right)\end{array}$ & $0,7-0,9$ & $30 \mathrm{~m}$ \\
\hline $\begin{array}{l}5\left(\mathrm{TM} \mathrm{e}^{\mathrm{e}}\right. \\
\left.\mathrm{ETM}^{+}\right)\end{array}$ & $1,5-1,7$ & $30 \mathrm{~m}$ \\
\hline 6 (TM) & $10,4-12,5$ & $120 \mathrm{~m}$ \\
\hline $6\left(\mathrm{ETM}^{+}\right)$ & $10,4-10,5$ & $60 \mathrm{~m}$ \\
\hline $\begin{array}{l}7\left(\mathrm{TM}_{\mathrm{e}} \mathrm{e}\right. \\
\left.\mathrm{ETM}^{+}\right)\end{array}$ & $2,0-2,3$ & $30 \mathrm{~m}$ \\
\hline $\begin{array}{l}8 \text { PAN } \\
\left(\mathrm{ETM}^{+}\right)\end{array}$ & $0,5-0,9$ & $15 \mathrm{~m}$ \\
\hline
\end{tabular}

Fonte: FLORENZANO (2002)

O estudo de WENG et al. (2004) procurou mostrar a viabilidade da utilização dos dados do canal 6 do satélite LANDSAT em pesquisas relacionadas à climatologia urbana, especificamente à extração de temperatura de superfície. Os autores utilizaram uma imagem LANDSAT ETM ${ }^{+} 7$ (canal 6) da cidade de Indianápolis (EUA) para investigar a variação das temperaturas das superfícies na cidade. O estudo chama atenção para o fato de ser a emissividade $(\varepsilon)$ das superfícies um importante elemento a ser considerado e corrigido.

Da mesma forma, NICHOL (1994) acredita que entre os parâmetros que contribuem para erros na derivação de temperaturas de superfície por meio de dados de radiância do satélite, aqueles que produzem relativas imprecisões entre os tipos de coberturas, tal como a emissividade, são importantes para monitorar variações de temperatura em áreas urbanas. 
Embora alguns autores estejam fazendo uso das imagens de satélite do sensor TM, canal 6, do satélite LANDSAT TM 5 para a estimativa de temperatura de superfície, vale lembrar que os resultados não podem proporcionar a expressão real do campo térmico.

Ressalta-se que as temperaturas derivadas do sensor não são idênticas à temperatura do ar medida na camada de ar próxima à superfície. Segundo Fialho e Azevedo (2009), a utilização de dados de satélites limita a estimativa das condições térmicas da cidade ao nível dos telhados, o que difere das observações intra-urbanas. Mesmo que se utilize a temperatura do ar ao mesmo tempo em que se obtém a imagem termal, os valores estimados de superfície servirão somente para a calibração da imagem, portanto, não é possível afirmar que a imagem termal equivale aos registros da temperatura do ar dentro da cidade.

Existem inúmeros modelos para a extração da temperatura aparente da superfície da Terra a partir das imagens originadas do satélite LANDSAT. BARIOU et al. (1993) sintetizaram três dessas técnicas, porém, ressaltaram que estas se apresentam como técnicas que subestimam a temperatura, pois não são feitas correções com relação aos efeitos atmosféricos e de emissividade. Uma delas é a de MARALET et al. (1985), que foi utilizada por TARIFA e ARMANI (2000) para a Região Metropolitana de São Paulo. De acordo com MARALET et al. (1985), a obtenção da temperatura de superfície é feita por meio da conversão do número digital (DN) de cada pixel da imagem do canal termal em temperatura aparente, com a utilização do seguinte modelo de regressão quadrática:

$$
T=209.831+0.834 D N-0.00133 D N^{2}
$$

A simples transformação das radiâncias (número digital) captadas pelo satélite em temperatura, no caso da equação acima, é feita supondo que a superfície observada é um corpo negro, cujo valor de emissividade é igual a 1. Todavia, uma superfície vegetada, por exemplo, não se comporta como um corpo negro perfeito, possuindo valores de emissividade sensivelmente menores que a unidade. Isso faz com que os valores de temperatura fornecidos pelo sensor sejam comprometidos pelo efeito da emissividade, que leva a uma subestimação do valor de temperatura de superfície. Assim, o valor de temperatura obtido deve ser chamado de "temperatura aparente" da superfície (SOBRINO et al., 1993) e só pode ser considerado como representativo da realidade terrestre, depois de ser corrigido quanto aos efeitos atmosféricos e de emissividade da superfície. Vale ressaltar que TARIFA e ARMANI (2000) tiveram - cuidado de utilizar o termo temperatura aparente para os mapas de temperatura elaborados a partir das estimativas pelo satélite.

Já a técnica apresentada por NICHOL (1994) e utilizada por COLLISCHONN (1998) para a Região Metropolitana de Porto Alegre, utiliza a equação inversa da lei de Planck e leva em consideração os efeitos da emissividade das superfícies, ou seja, esta técnica vai além da simples aplicação da lei de Planck, contudo, ainda assim, apresentou resultados limitados com relação a estimativa das temperaturas de superfície, pois não corrige os efeitos atmosféricos. A correção da emissividade é calculada conforme a seguinte equação: 
Onde:

$$
T s=\frac{T b}{1+\left(\frac{\lambda T b}{\alpha}\right) \cdot \ln \varepsilon}
$$

$T b$ = temperatura que emitiria a superfície se fosse um corpo negro perfeito;

$\lambda=$ comprimento de onda médio do infravermelho termal $=11,5 \mu \mathrm{m}$

$\alpha=\mathrm{hc} / \mathrm{K}=1.438 \times 10^{-2} \mathrm{~m} \mathrm{~K}$, onde:

$\mathrm{K}=$ constante de Stefan-Boltzmann $\left(1.38 \times 10^{-23} \mathrm{~J} / \mathrm{K}\right)$

$\mathrm{h}=$ constante de Planck $\left(6.28 \times 10^{-34} \mathrm{~J} / \mathrm{seg}\right)$

$\mathrm{c}=$ velocidade da luz $\left(2.998 \times 10^{8} \mathrm{~m} / \mathrm{seg}\right)$

$\ln \varepsilon=$ logaritmo natural da emissividade da superfície

Outra técnica, também bastante utilizada, é a de MARKHAM e BARKER (1986). A equação utilizada neste caso é mais complexa, pois envolve, além da emissividade, outros elementos tais como, a radiação e a transmissividade atmosféricas e por isso pode ser considerada de maior precisão, mesmo assim, LAYMON e QUATTROCHI (2004) ressaltam que o resultado ainda apresenta distorções. A temperatura de superfície, neste caso, é determinada por:

$$
T_{s}(\lambda)=\frac{C_{2}}{\ln \left(\left(\frac{C_{1}}{L_{s}(\lambda)}\right)+1\right)}
$$

Onde $C_{1}$ e $C_{2}$ são constantes de calibração iguais a $60.776 \mathrm{~mW} \mathrm{~cm}^{2}$ ster $^{1} \mu \mathrm{m}^{1}$ e $1260.56 \mathrm{~K}$, respectivamente. A radiação da superfície, $L_{s}(\lambda)$ pode ser expressa em termos da radiação observada, $L_{0}(\lambda)$, como:

$$
L_{s}(\lambda)=\frac{L_{0}(\lambda)-t\left(1-\varepsilon_{s}\right) L_{d}(\lambda)-L_{p}(\lambda)}{t \varepsilon_{s}}
$$

Onde:

$L_{0}(\lambda)=$ radiância espectral aparente no satélite na banda $\lambda$;

$L_{d}(\lambda)=$ radiação de ondas longas que atinge a superfície;

$L_{p}(\lambda)=$ radiação atmosférica;

$\varepsilon_{\mathrm{s}}=$ emissividade da superfície; e

$\mathrm{t}=$ transmissividade atmosférica.

\section{Área de estudo}

A área de estudo selecionada para testar o modelo diz respeito à bacia hidrográfica do Rio Monteiro. Localiza-se a nordeste do Distrito Federal e insere- 
se no Alto Curso da bacia hidrográfica do rio São Bartolomeu, sendo marcada por características antagônicas: por um lado a ocupação humana, representada pela cidade de Planaltina, por outro a presença de uma importante Unidade de Conservação - parte da Estação Ecológica de Águas Emendadas.

Com a inauguração de Brasília, toda a extensão territorial de Planaltina foi incorporada ao Distrito Federal passando, assim, a ser classificada como cidade-satélite que atendia às necessidades das populações excedentes do Plano Piloto. Desde então, tem-se observado um crescente aumento populacional e uma expansão da área urbana em direção à Estação Ecológica de Águas Emendadas e em direção oeste. Segundo dados do IBGE (2007), em 1960 a população de Planaltina era de 2.917 habitantes e, hoje, superando a marca dos 140.000 habitantes.

O clima da região, na classificação de Strahler, é classificado como Tropical Alternadamente Úmido e Seco, onde as chuvas se concentram no verão. O período chuvoso corresponde aos meses de outubro a abril e o período seco corresponde aos meses de maio a setembro, no inverno.

A variação da temperatura do ar está relacionada às variações altimétricas locais. Assim, admite-se que a temperatura para o mês mais frio seja superior a $18{ }^{\circ} \mathrm{C}$, característica encontrada nas regiões que se situam, aproximadamente, nas áreas com cotas altimétricas abaixo de $1000 \mathrm{~m}$, ou seja, ao longo do vale do rio Monteiro.

A área-teste é caracterizada, no aspecto fitogeográfico, pelo domínio morfológico dos Cerrados, podendo-se distinguir as seguintes tipologias campo: agregando-se ao campo limpo e ao campo antrópico; cerrado típico; e mata ciliar.

\section{PROCEDIMENTOS METODOLÓGICOS}

\section{Identificação da Cobertura do solo}

Segundo NOVO (1998), o termo Uso da Terra refere-se à utilização "cultural" da terra, enquanto que o termo Cobertura do Solo refere-se ao seu revestimento. Por exemplo: áreas florestadas que, embora sejam um só tipo sob o ponto de vista de cobertura, podem ter diferentes usos: lazer, exploração de madeira, reservas, etc. Sendo assim, ao se adotar um sistema de classificação de uso da terra, deve-se levar em conta um nível de detalhamento tal que não foi necessário nesta pesquisa específica, uma vez que o objetivo era identificar as principais coberturas e não seus usos específicos. Portanto, este plano de informação reuniu dados sobre a cobertura do espaço geográfico da bacia do Rio Monteiro em junho de 2003. Constitui-se de um mapa temático, onde cada classe identifica um tipo de cobertura, seja ele natural ou decorrente de atividades antrópicas.

O mapa de cobertura do solo foi elaborado por meio do processamento digital da imagem LANDSAT 5 TM, órbita ponto 221.71, do dia 11 de junho de 2003 utilizando a técnica de segmentação de imagens. Este método pode ser descrito como sendo o processo de divisão de uma imagem em segmentos não sobrepostos. A segmentação de imagens divide a imagem em grupos espectrais únicos e inclui um componente espacial. A exigência é que todos os pixels em um grupo (ou segmento) sejam espacialmente contíguos. Estes segmentos 
podem ser usados para extrair e classificar feições produzindo uma imagem melhor classificada. O programa de segmentação utilizado neste trabalho foi o RSAC (Remote Sensing Application Center) desenvolvido para o ERDASIMAGINE 8.5 (RUEFENACHT, et al., 2002).

Primeiramente, a partir da interpretação visual da imagem digital, foram identificadas as classes de cobertura preponderantes na cena. Depois de identificadas, optou-se por elaborar dois mapas de cobertura do solo, um com quatro classes: área urbana, área rural, área vegetada e água, neste caso as áreas vegetadas foram consideradas no todo; e outro com sete classes: área urbana, área rural, água, campo, cerrado, mata ciliar e reflorestamento, neste outro caso, as áreas vegetadas foram diferenciadas. Este procedimento foi realizado com o intuito de avaliar se a utilização de uma classificação mais refinada, para a estimativa da temperatura de superfície, possibilita um resultado mais preciso ou não.

\section{Estimativa da temperatura de superfície}

Os valores da temperatura de superfície foram obtidos por meio da técnica desenvolvida por Artis e Carnahan (apud NICHOL, 1994), e utilizada por COLLISCHONN (1998) para determinação das temperaturas de superfície na Região Metropolitana de Porto Alegre. Esta técnica utiliza equação (2), citada anteriormente.

A resolução da equação (2) foi definida em três passos: definição do numerador da equação, definição do denominador da equação e definição da temperatura da superfície. Utilizou-se, para tanto, o Sistema IDRISI ${ }^{13}$. O IDRISI é um software que reúne ferramentas nas áreas de processamento de imagens, sensoriamento remoto, SIG, geoestatística, apoio a tomada de decisão e análise de imagens geográficas de uma forma prática e de fácil uso. Vale salientar que a metodologia não deve, necessariamente, estar atrelada a um sistema específico, podendo ser desenvolvida por qualquer outro sistema de preferência do usuário.

O processamento digital das imagens utilizando-se o software IDRISI pode ser dividido em quatro operações básicas: restauração, realce da informação, criação de novos canais, e transformação e classificação das imagens. As ferramentas de análise geográfica utilizadas foram: THERMAL, RECLASS, ASSIGN, SCALAR e OVERLAY. RECLASS e ASSIGN são utilizados para isolar classes de interesse em uma imagem, bem como atribuir novos valores a estas classes. Os módulos SCALAR e OVERLAY produzem imagens novas como resultado de operações matemáticas sobre uma imagem ou entre duas imagens existentes.

\section{0 passo: Definição do numerador da equação (Tb)}

Para a estimativa da temperatura do corpo negro (Tb) de cada pixel da banda termal (canal 6) do satélite LANDSAT 5 TM, submeteu-se essa banda a um processo de conversão por meio do módulo THERMAL do Sistema IDRISI. A imagem de saída foi denominada de resultado 1: (Tb) temperatura do corpo negro.

${ }^{13}$ A metodologia seguida recomenda a utilização do software IDRISI para esta etapa, por isso, este foi utilizado nesta pesquisa, salienta-se que outros softwares também podem ser utilizados. 


\section{$2^{\circ}$ passo: Definição do denominador da equação}

A imagem resultado $1:$ (Tb) temperatura do corpo negro foi multiplicada pelo valor médio do comprimento de onda termal $(\lambda=11,5$ $\mu \mathrm{m})$ do módulo SCALAR. A imagem de saída foi denominada resultado 2 e representa a expressão $(\lambda T b)$.

A imagem resultado 2 ( $\lambda \mathbf{T} \mathbf{T})$ foi dividida pelo valor de $\alpha(0,01438)$, também por meio do módulo SCALAR. A imagem de saída foi denominada resultado $3(\lambda \operatorname{Tb} / \alpha)$.

O termo In $\boldsymbol{\varepsilon}$ foi obtido atribuindo-se os valores do logaritmo natural da emissividade de cada classe de cobertura do solo a cada pixel do mapa de cobertura. O módulo ASSIGN foi utilizado para tal atribuição. A imagem de saída foi denominada resultado 4: logaritmo da emissividade $(\ln \varepsilon)$.

Vale lembrar que a técnica foi testada utilizando-se dois mapas de cobertura de solo diferentes. Como já citado, um deles com quatro classes e outro com sete. Outro aspecto que merece ser comentado é fato de os valores de emissividade utilizados não terem sido coletados em campo, mas sim retirados da bibliografia. Dada a variação significativa da emissividade para as áreas vegetadas e superfícies construídas e, tendo em vista a importância deste parâmetro na obtenção dos valores de temperatura de superfície, realizou-se uma busca na bibliografia com o intuito de elaborar um quadro de valores de emissividade (quadro 1) e, a partir deste, optar pelos valores mais próximos da realidade da área de estudo. Dessa forma, optou-se por utilizar valores médios de emissividade para cada classe de cobertura do solo identificada.

Quadro 1: emissividades típicas de alguns materiais terrestres

\begin{tabular}{|l|l|l|}
\hline \multicolumn{1}{|c|}{ MARTERIAL } & FONTE & \multicolumn{1}{c|}{$\begin{array}{c}\text { VALOR DE } \\
\text { EMISSIVIDADE }\end{array}$} \\
\hline Água limpa & MENESES (2001) & $0,98-0,99$ \\
\hline Neve úmida & MENESES (2001) & $0,98-0,99$ \\
\hline Pele úmida & MENESES (2001) & $0,97-0,99$ \\
\hline Gelo seco & MENESES (2001) & $0,97-0,98$ \\
\hline Áreas vegetadas & NICHOL (1994) & 0,95 \\
\hline Vegetação verde & MENESES (2001) & $0,96-0,99$ \\
\hline Vegetação seca & MENESES (2001) & $0,88-0,94$ \\
\hline Vegetação com copa fechada & COLLISCHONN (1998) & $0,97-0,98$ \\
\hline Vegetação mais aberta & COLLISCHONN (1998) & 0,96 \\
\hline Culturas variadas & COLLISCHONN (1998) & $0,93-0,97$ \\
\hline Espaços verdes urbanos & COLLISCHONN (1998) & 0,90 \\
\hline Solo úmido & MENESES (2001) & $0,95-0,98$ \\
\hline Solo seco & COLLISCHONN (1998) & 0,91 \\
\hline Solo mineral seco & MENESES (2001) & $0,92-0,94$ \\
\hline Áreas não vegetadas & NICHOL (1994) & 0,92 \\
\hline Basalto & MENESES (2001) & $0,92-0,96$ \\
\hline Asfalto & MENESES (2001) & $0,94-0,97$ \\
\hline Concreto & COLLISCHONN (1998) & 0,97 \\
\hline Granito & MENESES (2001) & $0,83-0,87$ \\
\hline Metal polido & MENESES (2001) & $0,16-0,21$ \\
\hline Alumínio & MENESES (2001) & $0,03-0,07$ \\
\hline
\end{tabular}


A partir desse quadro foram selecionados os valores médios para cada classe de cobertura do solo definida nas imagens, a saber: área urbana: 0,92; área rural: 0,94; água: 0,97; vegetação (considerada primeiramente como uma única classe):0,95; campo: 0,93; cerrado: 0,96; mata ciliar: 0,98 e reflorestamento: 0,99. Destaca-se que esses valores médios podem mascarar alguns resultados, uma vez que as condições ambientais podem modificar os valores de emissividade.

O termo $(\boldsymbol{\lambda} \mathbf{T b} / \boldsymbol{\alpha})$. In $\boldsymbol{\varepsilon}$ foi definido multiplicando-se a imagem resultado 3: $(\lambda \mathrm{Tb} / \alpha)$ pela imagem resultado 4: logaritmo da emissividade por meio do módulo OVERLAY. A imagem de saída foi denominada resultado 5: $(\lambda \operatorname{Tb} / \alpha) \cdot \ln \varepsilon$.

Para obter-se uma imagem onde cada pixel tivesse o valor 1 definiu-se uma nova imagem, à qual atribuiu-se o valor inicial 1 para todos os pixels da imagem criando-se uma nova imagem (pixel valor 1) com o módulo INITIAL. Assim, o denominador da equação (resultado 6), foi encontrado somando-se, por meio do módulo OVERLAY, as imagens resultado 5 e pixel valor 1.

\section{$3^{\circ}$ PASSO: Definição da temperatura da superfície e elaboração da imagem termográfica.}

Utilizando-se o módulo OVERLAY, dividiu-se a imagem resultado 1: (Tb) temperatura do corpo negro pela imagem resultado 6: $(1+(\lambda \mathrm{Tb} / \alpha)$. $\ln \boldsymbol{\varepsilon}$ ) e obteve-se a imagem resultado final, que corresponde à temperatura de superfície de cada pixel em graus Kelvin ( ${ }^{\circ} \mathrm{K}$ ).

As temperaturas resultantes foram, então, convertidas para graus Celcius $\left({ }^{\circ} \mathrm{C}\right)$ subtraindo-se o valor da temperatura do ponto de congelamento da água ao nível do mar, ou seja, 273,15 K, com o módulo SCALAR. Esta nova imagem foi submetida a uma reclassificação através do módulo RECLASS definindo-se as seguintes classes: Classe 1: menor que $15^{\circ} \mathrm{C}$; Classe 2: $15^{\circ} \mathrm{C}$ a $17^{\circ} \mathrm{C}$; Classe 3: $17^{\circ} \mathrm{C}$ a $19{ }^{\circ} \mathrm{C}$; Classe 4: $19^{\circ} \mathrm{C}$ a $21^{\circ} \mathrm{C}$; Casse 5: $21^{\circ} \mathrm{C}$ a $23^{\circ} \mathrm{C}$; Classe 6: $23^{\circ} \mathrm{C}$ a $25^{\circ} \mathrm{C}$; Classe 7: $25^{\circ} \mathrm{C}$ a $27^{\circ} \mathrm{C}$; e Classe 8: maior que $27^{\circ} \mathrm{C}$.

Para um melhor entendimento dos procedimentos adotados ver 0 fluxograma esquemático (Figura 1). 


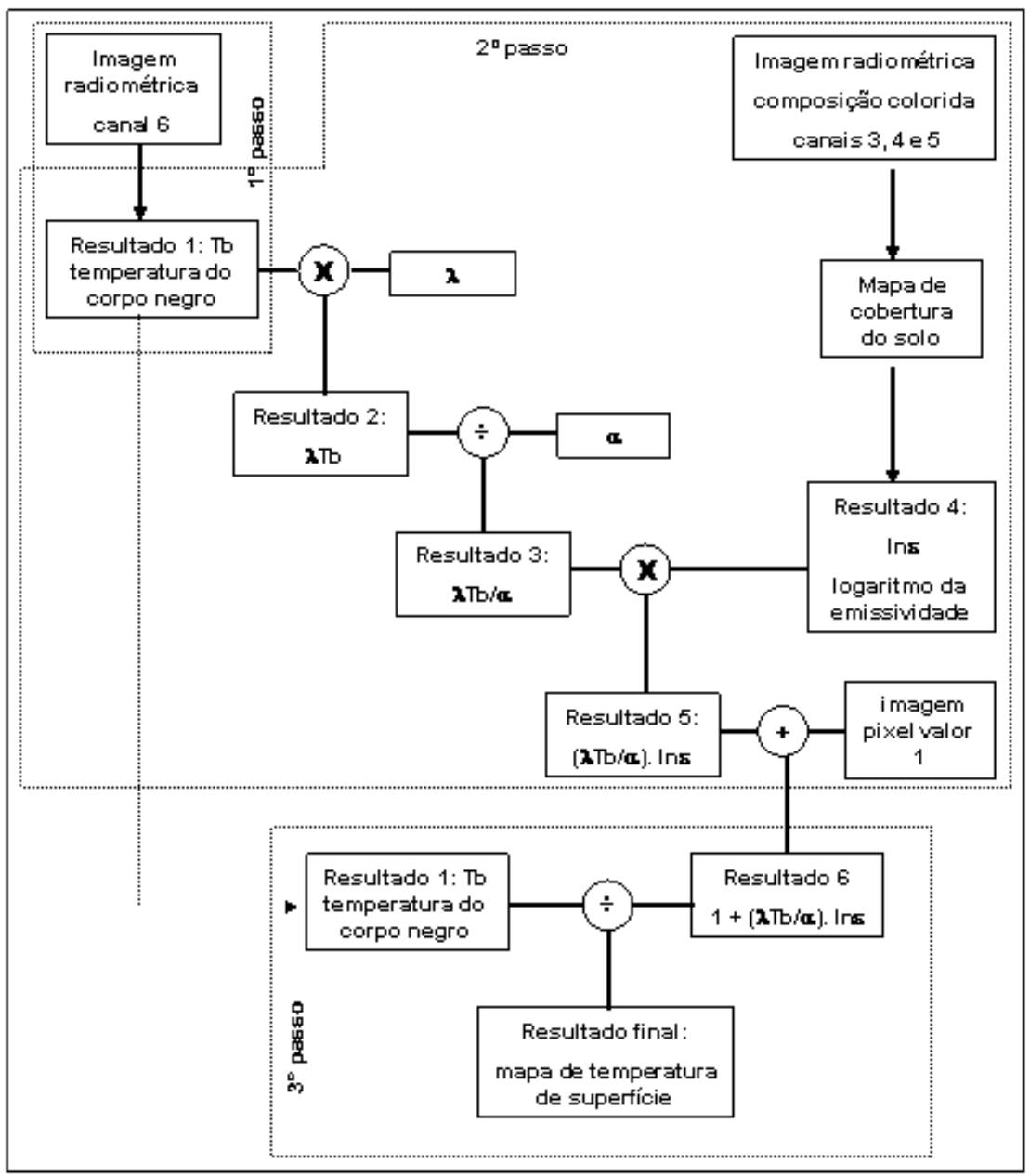

Figura 1: Fluxograma esquemático das operações matemáticas utilizadas para a identificação da temperatura de superfície.

\section{RESULTADOS E DISCUSSÃO}

Como já citado foram elaborados dois mapas de cobertura do solo em função da identificação de diferentes classes. A primeira classificação levou em consideração os grupos de cobertura mais gerais, onde a vegetação foi aglutinada em uma única classe assim como a área urbana (figura 2). Utilizando-se esta classificação e aplicando-se a equação (2) de estimativa de temperatura de superfície observou-se que o mapa resultante (figura 3) coloca em evidência o comportamento térmico das principais coberturas do solo. Os contrastes térmicos principais aparecem entre as áreas cobertas de água, vegetação, espaços rurais e urbanos.

Já o mapa de cobertura do solo gerado com base na classificação mais detalhada (Água, Cerrado, Campo, Mata Ciliar, Reflorestamento, Área Rural, Área Urbana Consolidada e Área Urbana em Consolidação) (Figura 4) mostrou um resultado mais aprimorado em relação aos contrastes térmicos (Figura 5). 
Esta divisão em um maior número de classes é significativa no momento em que representa um maior número de alvos, os quais possuem valores de emissividade diferenciados daqueles admitidos para uma classe mais geral.

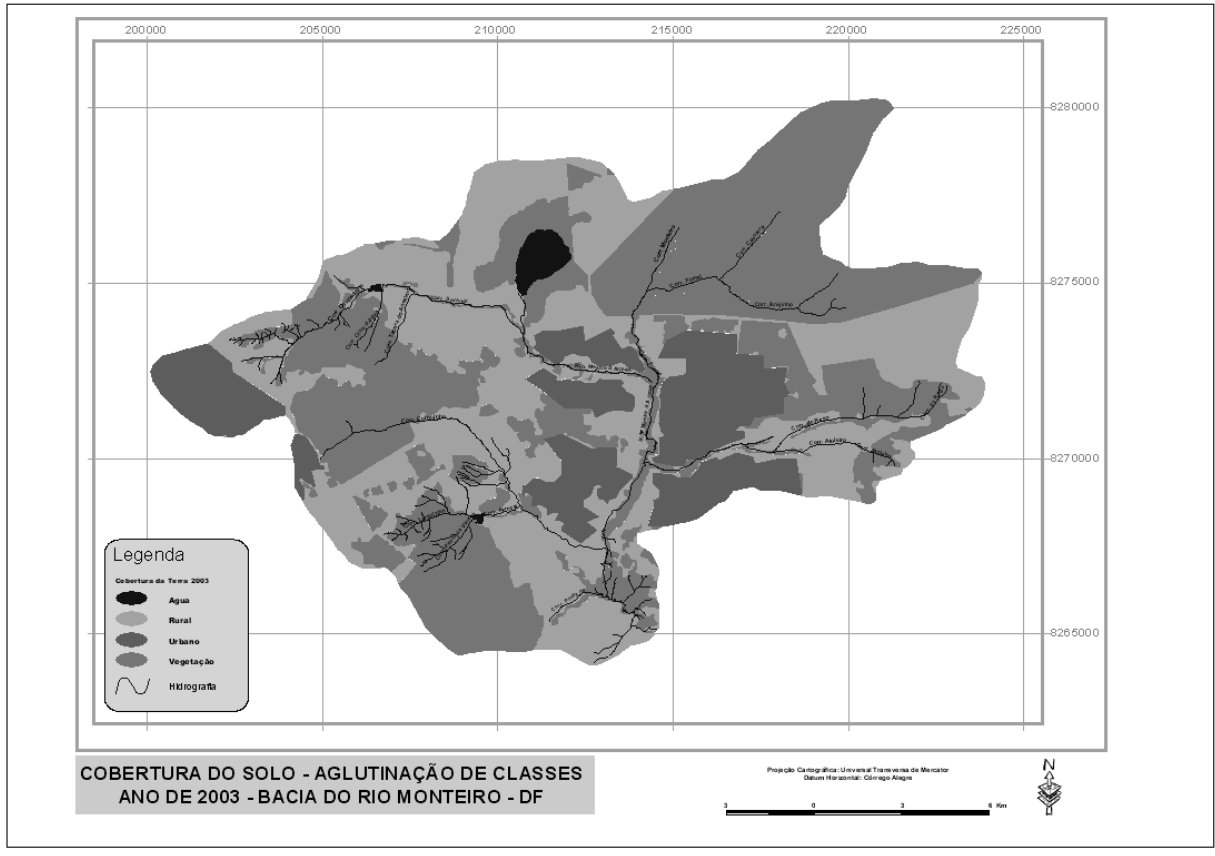

Figura 2: Mapa de distribuição das classes de cobertura do solo no ano de 2003, na bacia hidrográfica do rio Monteiro - DF.

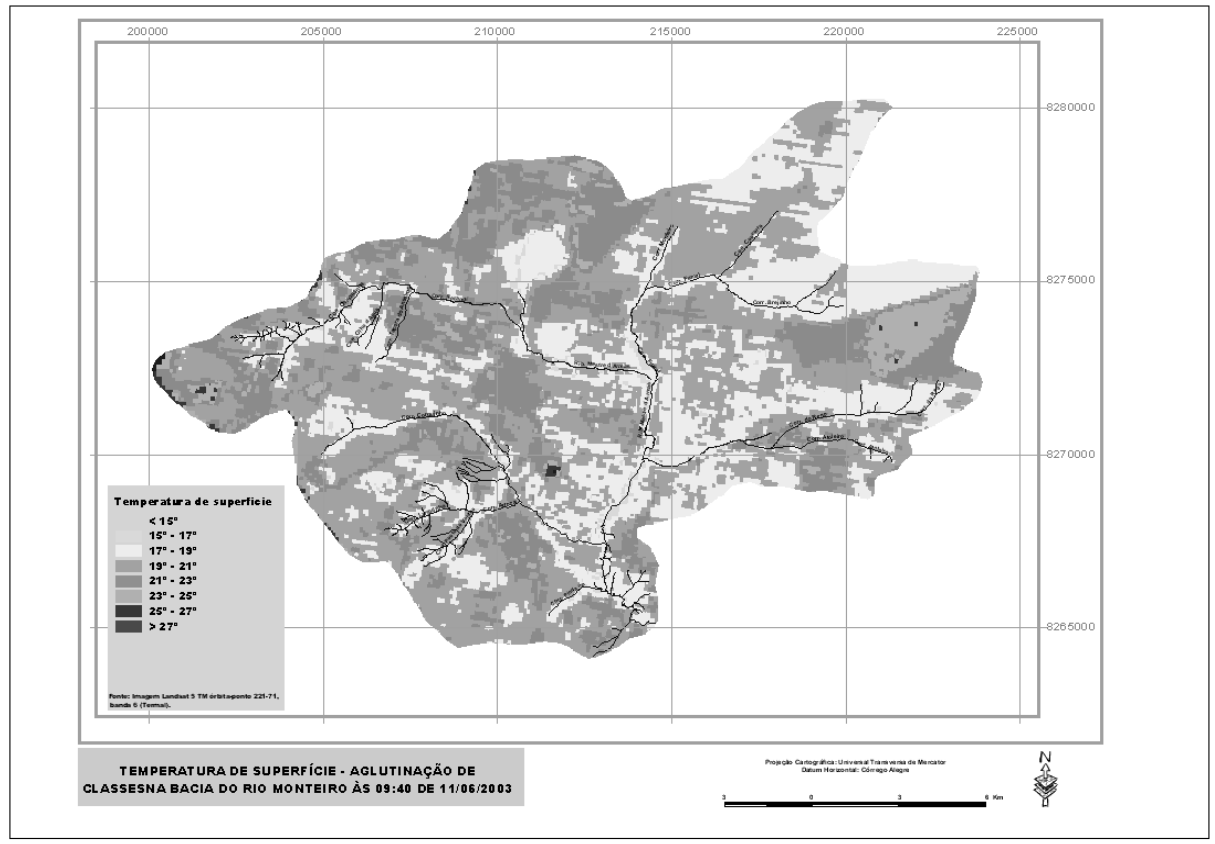

Figura 3: Mapa de distribuição da temperatura de superfície. 


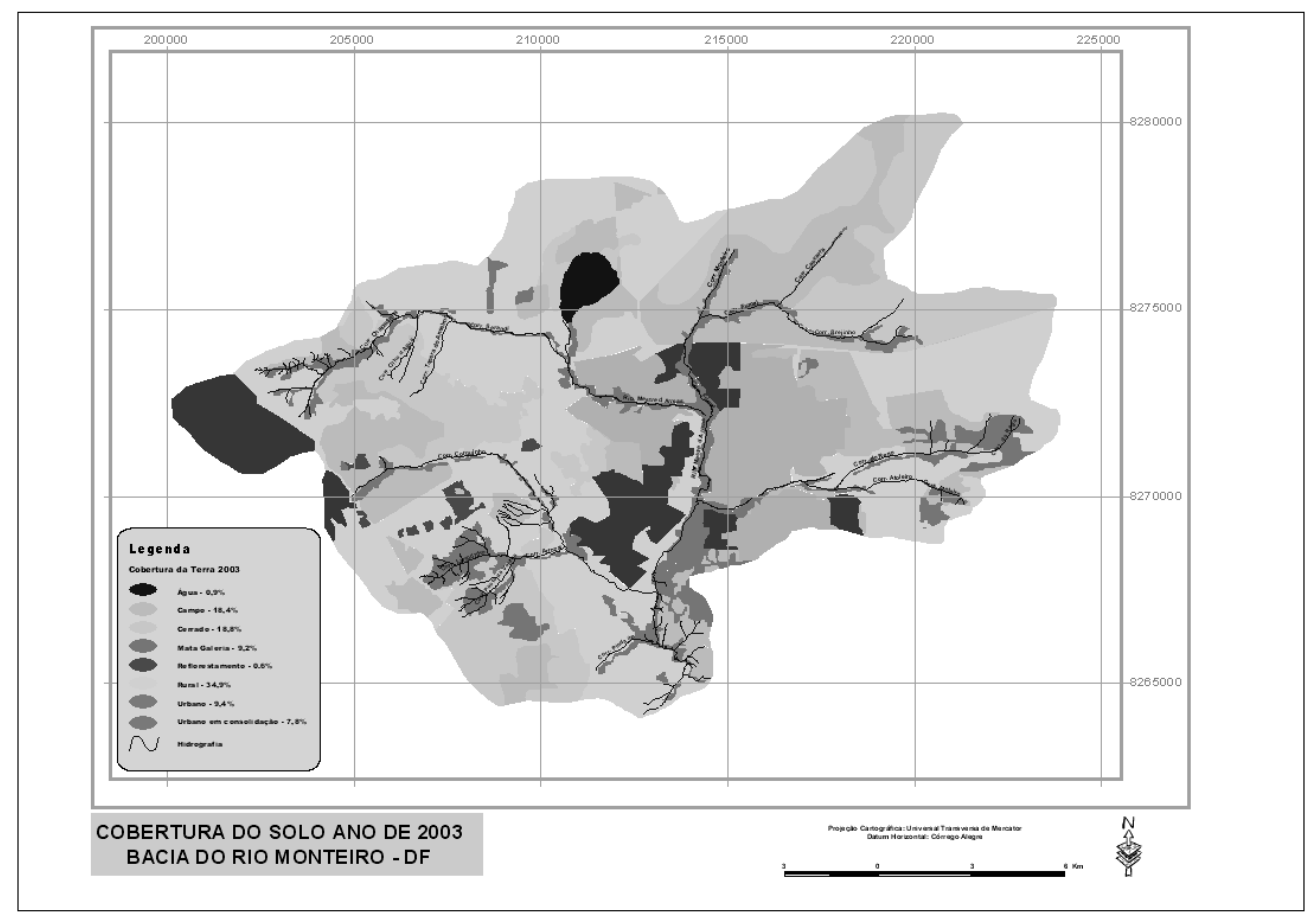

Figura 4: Mapa de distribuição das classes de cobertura do solo no ano de 2003, na bacia hidrográfica do rio Monteiro - DF.

Comparando-se os dois mapas de temperatura de superfície (Figuras 3 e 5) pode-se verificar que a espacialização dos dados de temperatura de superfície inferidos a partir dos valores de pixel, comportam-se diferentemente em relação ao maior detalhamento da identificação dos tipos de cobertura do solo. Aparentemente, quando se mantém uma classificação geral, aglutinandose classes de cobertura do solo, os valores de emissividade dos alvos (que já são valores médios) interferem diretamente na distribuição dos valores de temperatura de superfície, produzindo um novo arranjo na espacialização de dados sobre a temperatura de superfície.

Este novo arranjo pode estar relacionado com o efeito do distanciamento das bordas, sendo mais acentuado quanto mais circular a feição geométrica em análise.

No caso de bacias hidrográficas, o impacto das diferenças na temperatura de superfície sobre os processos atmosféricos e destes, sobre a dinâmica de recarga dos aqüíferos pode ser uma realidade que venha a merecer atenção, uma vez que o clima, por ser um dos mais importantes componentes do ambiente natural, pode se caracterizar como indicador de degradação ambiental. Esta possível funcionalidade é decorrente do fato de que ele afeta os processos geomorfológicos atuais, os da transformação dos solos, o crescimento e o desenvolvimento da vegetação e consequentemente, os processos hidrológicos que envolvem a dinâmica de uma bacia hidrográfica.

De acordo com as diferenças observadas nos mapas de temperatura de superfície, quando comparadas ao mapa de cobertura do solo, constata-se que os primeiros apresentam maior detalhamento e variância interna que os últimos. Além disso, os resultados da distribuição das temperaturas de 
superfície podem sugerir a necessidade de proteger prioritariamente as regiões das bacias junto às nascentes e divisores de águas, visto que extensas áreas rurais, mesmo que não tenham a mesma magnitude de impacto que uma ocupação urbana, ainda assim produzem áreas nucleares de calor (ligeira elevação de temperatura) em suas áreas centrais, com desdobramentos sobre o ciclo hidrológico local e sub-regional.

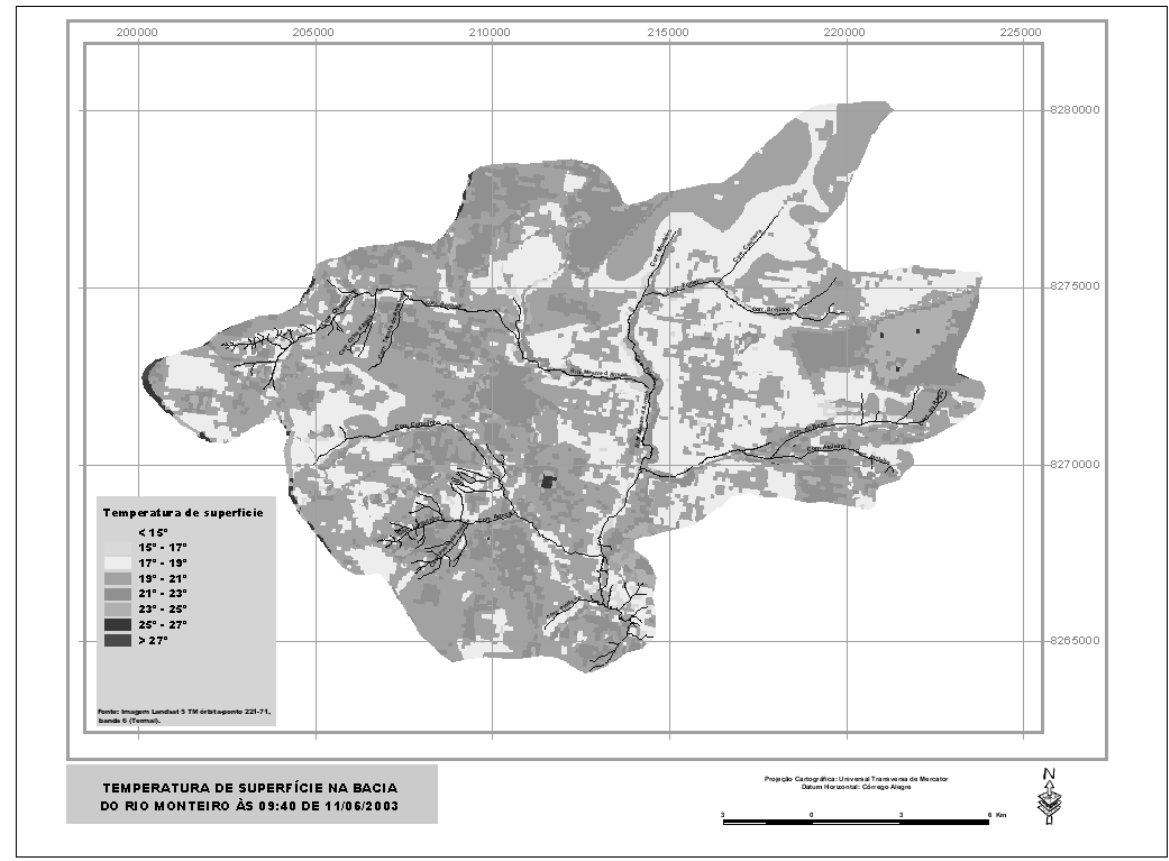

Figura 5: Mapa de distribuição da temperatura de superfície

Observa-se que a maior preocupação dos estudos envolvendo problemas em recursos hídricos têm-se voltado para a identificação de impactos acarretados em função de alterações na própria água ou na região adjacente ao corpo d'água, utilizando-se para a análise mapas de uso da terra. A inclusão de variáveis climáticas e suas alterações como promovedoras de impactos ambientais nos recursos hídricos é recente, contudo, o interesse pelo assunto vem crescendo e já existe, na bibliografia, uma gama de trabalhos referente ao tema.

A questão climática e seus efeitos, tanto em nível global como regional e local, vem sendo estudada com especial interesse pelos que se dedicam às ciências da natureza em todo mundo. O interesse na questão climática deve-se à sua importância fundamental para as atividades humanas, e por ser um dos poucos componentes do sistema ambiental sobre o qual o homem tem pouco ou nenhum poder de controle. Sobre isso SANTOS (1995) afirma que:

Atualmente, há tendência em não mais enfocar o clima como um fator de adversidade, negligenciando-o ou desprezando-o como recurso. Verifica-se uma valorização do clima pautado na consideração de que a exploração demográfica e o conseqüente aumento do consumo, com aumento da necessidade de alimentos, exercem demanda cada vez maior na capacidade de nosso planeta em manter a vida. Sob este 
aspecto, o clima em conjunto com o solo, leva a uma conjugação dinâmica no estabelecimento de condições dos "sistemas vitais" à sobrevivência do homem". SANTOS (1995)

No caso específico deste trabalho procurou-se mostrar, por meio de uma metodologia diferenciada das usualmente utilizadas, que a análise de variáveis climáticas pode contribuir para se determinar problemas atuais e futuros na dinâmica de uma bacia hidrográfica. Não se pode esquecer que a metodologia utilizada apresenta limitações, porém, pode oferecer informações que auxiliem na avaliação dos recursos naturais. Uma vez que a técnica seja aprimorada, estas informações serão, cada vez mais, essenciais para a gestão do território.

\section{CONSIDERAÇÕES FINAIS}

As estimativas de temperatura de superfície a partir do sensoriamento remoto termal utilizando-se imagens provenientes do satélite LANDSAT TM 5 são muito mais complexas do que o método aqui apresentado. Fatores como a natureza dos diferentes alvos, emissividade, efeitos atmosféricos, entre outros, contribuem para as dificuldades em determinar as temperaturas de superfícies em diferentes escalas espaciais. QUATTROCHI e GOEL (1995) destacam que, para se obter qualidade razoável nas estimativas, devem ser considerados vários processos de correção, entre eles, efeitos atmosféricos e de emissividade da superfície. Neste estudo não foram levados em consideração os efeitos atmosféricos, o que introduz um erro de alguns graus centígrados na resposta. Entretanto, a variação pode não ter sido muito elevada, pois a imagem utilizada neste estudo cobre uma pequena área e foi gerada em um dia com tempo estável.

Com relação à aplicação desta metodologia para a gestão de bacias hidrográficas observou-se que, para que os resultados encontrados possam servir de subsídio ao gerenciamento, novas pesquisas devem ser desenvolvidas, no sentido de confirmá-los utilizando-se de metodologias de campo, tais como a tomada da emissividade dos alvos com radiômetros (para melhorar a estimativa da temperatura de superfície) e o levantamento de dados hidrometeorológicos numa série temporal que permita a identificação de alterações nas variáveis hidrológicas.

\section{REFERÊNCIAS BIBLIOGRÁFICAS}

ALMEIDA, C. A. S. de. Estimativa de temperatura de superfície cultivada com trigo (Triticum aestivum L.), usando NOAA -14/AVHRR. Campinas, 1996. $101 \mathrm{f}$. Dissertação (Mestrado). Faculdade de Engenharia Agrícola, Universidade Estadual de Campinas.

BARIOU, R.; LECAMUS, D.; LE HENAFF, F. L'etalonnage des données. Rennes: Centre Regional de télédétection/UHB/Rennes 2, 1993.

COLLISCHONN, E. O campo térmico da região metropolitana de Porto Alegre: uma análise a partir da interação das variáveis ambientais na definição do clima local. Florianópolis, 1996, 152 f. Dissertação (Mestrado) Departamento de Geociências Universidade Federal de Santa Catarina. 
DOUSSET, B. and GOURMELON, F. Satellite multisensor data analysis of urban surface temperatures and landcover. Photogrammetric and Remote Sensing, n. 58, p. 43-54. 2003.

EHRLICH, D.; ESTES, J. E. e SINGH, A. Aplications of NOAA - AVHRR $1 \mathrm{~km}$ data for environmental monitoring. International Journal of Remote Sensing, v. 15, n. 1, p. 145 - 161. 1994.

FERREIRA, A. G. Interpretação de imagens de satélites meteorológicos: uma visão prática e operacional do hemisfério sul. Brasília: Stilo, 2002.

FIALHO, E. S.; AZEVEDO, T. R. de. Refletindo sobre o conceito de ilha de calor. In: Simpósio Brasileiro de Geografia Física Aplicada, 13, 2009, Viçosa. Anais... Viçosa: UfV. 1 CD ROM. DISPONÍVEL EM:

http://www.geo.ufv.br/simposio/simposio/trabalhos/resumos_expandidos/eixo8/008.pdf Acesso em dezembro de 2009.

FLORENZANO, T. G. Imagens de satélite para estudos ambientais. São Paulo: Oficina de Textos, 2002.

IBGE. Contagem da População 2007 . Rio de Janeiro: IBGE, 2007.

KERR, Y. H.; LAGOUARDE, J. P. e IMBERNON, J. Accurate land surface temperature retrieval from AVHRR data with use an improved split window algorithm. Remote Sensing of Environment. V. 41. P. 197 - 209. 1992.

KERR, Y. H.; LAGOUARDE, J. P.; NERRY, F. e OTTLÉ, C. Land surface temperature retrieval techniques and applications. In: QUATTROCHI, D. A e LUVALL, J. C. Thermal remote sensing in land surface processes. Boca Raton: CRC Press, 33 - 109, 2003.

LOMBARDO, M. A. Ilhas de calor nas metrópoles: o exemplo de São Paulo. HUCITEC, 1985.

MACHADO, H. M.; LAMPARELLI, R, A. C.; ROCHA, J. V. e ZULLO JUNIOR, J. Estudo da variação de parâmetros atmosféricos necessários para a correção atmosférica de imagens de satélite. In: SIMPÓSIO BRASILEIRO DE SENSORIAMENTO REMOTO, 11., 2003, Belo Horizonte. Anais... Belo Horizonte: INPE, 2003. p. 2537 - 2539. 1 CDROM.

MARALET, E.; BARTOLUCCI, L. A., LOZANO, D. F.; ANUTA, P. E. and McGILLEN, C. D. LANDSAT 4 and LANDSAT 5 thematic mapper data quality analysis, Photogrammetric Engineering and Remote Sensing, v. 51, n. 9:, p. 1407-1416. 1985.

MENDONÇA, F. O estudo do clima urbano no Brasil: evolução, tendências e alguns desafios. In: MONTEIRO, C, A. de F. e MENDONÇA, F. (org.) Clima urbano. São Paulo: Contexto, 2003. P. 175 a 192.

MENDONÇA, F; DUBREUIL, V. Termografia de superfície e temperatura do ar na RMC (região metropolitana de curitiba/PR). R. RA'E GA. n. 9, p. 25-35, 2005.

MENESES, P. R. Fundamentos de Sensoriamento Remoto. Texto Universitário do Curso de especialização em geoprocessamento aplicado ao zoneamento ambiental. Brasília: UnB/IG/LSR. 2001. 
MIRALLES, J. M. Fundamentos físicos de la teledetección: leyes e principios básicos. In: GANDIA, $S$ y MELIÁ, J. La teledetección en el seguimiento de los fenómenos naturales. Valéncia: Universitat de Valéncia, 1991. P. 50-83.

NICHOL, J. E. A GIS-based approach to microclimate monitoring in Singapore's high-rise housing estates. Photogrammetric Engineering \& Remote Sensing, v. 60, n. 10, p. 1225 - 1232. 1994.

NOVO, E. M. L de M. Sensoriamento Remoto: princípios e aplicações. São Paulo: Edgard Blücher, 1998.

QUATTROCHI, D. A. e GOEL, N. S. Spatial and temporal scaling of termal remote sensing data. Remote Sensing Reviews, v. 12, p. 255-286. 1995.

PETKOV, L.; PIERI, M.; MASELLI, F.; MARACCHI, G. Study and modelling of temperature spatial variability by NOAA - AVHRR thermal imagery. Photogrammetric Engineering \& Remote Sensing, v. 51, p.127-136. 1996.

PRICE, J. C. Estimating surfaces temperatures from satellite thermal infrared data - a simple formulation for the atmospheric effect. Remote Sensing of Environment, n. 13, p. 353 - 361. 1983.

ROTH, M.; OKE, T. R. and EMERY, W. J. Satellite derived urban heat islands from three coastal cities and the utilization of such data in urban climatology. International Journal of Remote Sensing, v.10, n. 11, p. 1699-1720. 1989.

RUEFENACHT, B. et al. (2002). New Technique for Segmenting Images. Washington, DC: United States Department of Agriculture. Disponível em <http://www.usda.gov.> Acesso em: 15 mai. 2003.

SANTOS, M. J. Z. Bioclimatologia e Geografia. Rio Claro: DG/IGCE/UNESP, 1995.

SILVA, J. de F. da e D'ANGIOLELLA. A Climatologia aplicada na gestão de sistemas hidrológicos. Meio Ambiente, n. 01, p. 40-45, jan/fev, 2002.

SOBRINO, J. A.; COLL, C. e CASELLES, V. La medida de la temperatura desde satélites. In: La teledetección en el seguimiento frf lod fenómenos naturales. Climatologia y desertificación. Valéncia: Facultat de Física, Universitat de Valéncia, 1993. P. 153 181.

THOMAS, A.; BYRNE, D and WEATHERBEE, R. Coastal sea surface temperature variability from LANDSAT infrared data. Remote Sensing of Environment, n. 81, p. $262-272$. 2002.

WENG, Q.; DENGSHENG, L. and SCHBRING, J. Estimationa of land surface temperaturevegetation abundance relationship for urban heat island. Remote Sensing of Environment, v. 89, p. 467-483. 2004.

ZULLO JUNIOR, J. Correção atmosférica de imagens de satélite e aplicações. Campinas, 1994. 189 f. Tese (Doutorado). Faculdade de Engenharia Elétrica, Universidade Estadual de Campinas. 